\title{
DNA Methylation and Telomerase Linked Mechanisms Potentially Underlie the Effects of Bisphenol A and Its Analogues (Bisphenol $F$ and Bisphenol S) in Breast Cancer Cells
}

\author{
Nathalie Zgheib ${ }^{1}$, Zainab Awada1 ${ }^{1}$ Reem Akika ${ }^{1}$, Rihab Nasr ${ }^{1}$, Akram Ghantous $^{2}$ \\ ${ }^{I}$ Department of Pharmacology and Toxicology, American University of Beirut, Lebanon, ${ }^{2}$ International Agency for \\ Research on Cancer, France
}

Background: Bisphenol A (BPA), an estrogen-like endocrine disruptor, has been associated with breast carcinogenesis in multiple studies, so plastic manufacturers shifted towards the less-studied and potentially less toxic BPA analogues, bisphenol F (BPF) and bisphenol S (BPS). Estrogen receptor (ER) is involved in the upregulation of global DNA methylation and telomerase expression, which have been associated with breast cancer development. However, the role of endocrine disruptors in the regulation of DNA methylation and telomerase expression has not been fully elucidated.

Aims: We aimed to evaluate the metabolic activity, viability and mobility, as well as the DNA methylation and telomeraselinked mechanisms of BPA and its analogues (BPF and BPS) on breast cancer cell lines (ER positive: MCF-7 and ER negative: MDA-MB-231).

Methods: We assessed metabolic activity, viability and mobility of MCF-7 and MDA-MB-231 cells after treatment with BPA, BPF and BPS with/without ER inhibitor. At day 1 of treatment of MCF-7 cells, RNA expression of genes involved in DNA methylation (DNMT1, DNMT3a, DNMT3b) and demethylation (APOBEC3B and TET 2 and 3 enzymes) was quantified. Telomerase expression and relative telomere length (RTL) were also evaluated. Cell invasion assay and LINE1 methylation (as a surrogate marker for global methylation) are in progress for both cell lines. Similar assays will be performed on normal-like breast epithelial cells.

Results: In MCF-7 cells, BPA, BPF and BPS caused a dose- and ER-dependent increase in cell metabolic activity and viability, with BPS being 10 times less potent than BPA and BPF. All three endocrine disruptors increased MCF-7 cell mobility over 24 hours. As expected, MDA-MB-231 cells, being ER negative, did not show changes in these parameters. In MCF-7 cells, the three endocrine disruptors increased the expression of DNMT1 and telomerase (but without affecting RTL); TET2 expression was increased by both BPA and BPS while TET3 expression was increased by only BPA. Similarly to the phenotypic changes, these molecular alterations were ER-dependent (Figure1).

Conclusions: DNA methylation and telomerase are potentially involved in the effects of BPA and its analogues on MCF-7 cells. Our results hopefully set the basis for studies aiming to evaluate the mechanism of endocrine disruptors. 